\title{
Speed and sense-data: Understanding the senses as tensors
}

\author{
Rafael Duarte Oliveira Venancio ${ }^{1}$
}

\begin{abstract}
This paper discuss the problem of motion within sense-data concept. Using the sense of speed as starting-point, we debate how it is possible to find a conceptual formulation that combines the idea of mental states with its physicalist criticism. The answer lies in the field of quantum mechanics and its concept of tensor, a geometric object that has a mathematical matrix representation. Thinking about examples taken from the car racing world, where the sense of speed is preponderant, we see how the mental condition of speed is represented matrix-like, tensor-like, rather than ephemerally as the more traditional sense-data formulation advocates.
\end{abstract}

Keywords: Sense-data; Speed; Tensors

In a well-known formulation, G. E. Moore defines sense-data as "that kind of knowledge, which we should call knowledge by means of the senses". ${ }^{2}$ These senses are usually reduced to the sense of sight, and the analysis focuses for example on the notions of color, size and shape. ${ }^{3}$

Among the various criticisms of the concept, John T. Kearns show us that one of them "appears to be movement [...], sense-data don't move". ${ }^{4}$ However, must we consider that there is a dead end situation to sense-data formulation just because there is no way to deal with senses beyond its ephemeral condition?

In his critique of Moore, Kearns seems to concentrate on the chosen senses as examples in the formulation of the concept, forgetting the common sense definition that the concept has among all the authors who use it. "On the most common conception, sense data [...] have three defining characteristics: (i) Sense data are the kind of thing we are directly aware of in perception; (ii) Sense data are dependent on the mind; and (iii) Sense data have the properties that perceptually appear to us". 5

Thinking about these three characteristics, we can see that motion has what another sense data have, even if the motion leads to the indeterminacy of the classic sense data of color and shape. After all, "Hardin discusses psychological experiments that seem to demonstrate indeterminacy of color and shape appearances: in some cases, subjects

\footnotetext{
${ }^{1}$ Rafael Duarte Oliveira Venancio, PhD, is professor at Universidade Federal de Uberlândia, Brazil.

${ }^{2}$ Moore, G. E. (1993). Sense-data. In Selected writings. London: Routledge, p. 45.

${ }^{3}$ lbid, p. 48

${ }^{4}$ Kearns, J. T. (1996). Reconceiving experience: a solution to a problem inherited from Descartes. Albany: State University of New York Press, p. 79.

${ }^{5}$ Huemer, Michael, "Sense-Data", The Stanford Encyclopedia of Philosophy (Spring 2011 Edition), Edward N. Zalta (ed.), URL = <https://plato.stanford.edu/archives/spr2011/entries/sense-data/>.
} 
can visually detect the existence of an object without being able to make out any apparent color, can detect motion without awareness of the shape or color of the moving object". 6

In this way, it seems clear to us that we should not speak of motion itself, but seek a definition of a sense of motion. Our proposal is that speed is that sense.

We all know that speed is a scalar quantity, having the dimensions of distance divided by time. But it is not na easy concept in Physics. Richard Feynman points out that "even though we know roughly what 'speed' means, there are still some rather deep subtleties; consider that the learned Greeks were never able to adequately describe problems involving velocity. The subtlety comes when we try to comprehend exactly what is meant by 'speed'"?

Feynman explains that "in order to get the speed more and more accurately, we should take a smaller and smaller time interval [...]. The foregoing definition involves a new idea, an idea that was not available to the Greeks in a general form. That idea was to take an infinitesimal distance and the corresponding infinitesimal time, form the ratio, and watch what happens to that ratio as the time that we use gets smaller and smaller and smaller. [It] is the beginning of a new branch of mathematics, called the differential calculus". 8

Therefore, Feynman builds the argument that show us speed as a derivative. But we must point out that the sense of speed must not be confused with the measure of speed. Even to understand mathematically all the implications of speed as a scalar quantity we must view it geometrically, or, as a quantum mechanics proposes, we must view speed as a tensor.

Tensor is a geometrical object that describes, forming a matrix, linear relations between vectors, scalars and even other tensors. Feynman says that "The mathematics of tensors is particularly useful for describing properties of substances which vary in direction-although that's only one example of their use. Since most of you are not going to become physicists, but are going to go into the real world, where things depend severely upon direction, sooner or later you will need to use tensors". 9

At this point of reasoning, we can then consider that the sense of speed can be understood as a tensor as well. This seems to be in consonance with the reports and studies about the mental perception of racing drivers. In Don Alexander's book about NASCAR drivers' techniques ${ }^{10}$, for example, he shows that "finding more speed" is a mix, built inside driver's mind, between infinitesimal calculus about distance and time in a corner with the notion of cornering line. All the concepts exist in mind only because in track there is only the car and the driver that controls it.

\footnotetext{
${ }^{6}$ Ibid.

${ }^{7}$ Feynman, R. P., Leighton, R. B., \& Sands, M. L. (1963). The Feynman lectures on Physics (Vol. 1). Pasadena, CA: California Institute of Technology, i.8.

${ }^{8}$ Ibid.

${ }^{9}$ Feynman, R. P., Leighton, R. B., \& Sands, M. L. (1963). The Feynman lectures on Physics (Vol. 2).

Pasadena, CA: California Institute of Technology, i.31.

${ }^{10}$ Alexander, D. (2001). Stock car driving techniques. St. Paul, MN: MBI.
} 
This matrix-line reasoning is common among racing drivers and racing fans, being widespread in books, interviews and even videogames. Therefore, if the sense of speed is represented as a matrix, it give us a powerful insight.

Sense data, normally, is refuted by physicalism. "Physicalists believe that the world is entirely physical; in particular, they believe that mental states either do not exist or are reducible to physical states" $"$. But if we start to understand the mental states within an inspirational corpus of physical analysis - like tensors - we can build a "matrix bridge" between the "mind world" of sense-data, the "physical world" and the "real world" that Feynman mentions.

Tensors are a powerful concept not only for its geometrical features, but also because its ad infinitum reasoning that is so peculiar in quantum mechanics. Sense data need to be seen now as abysmal and non-ephemeral structures. Matrices and not singular data. A single sense datum is a relation of several sense data that composes it in a matricial way.

This is the powerful insight that the world of speed, described by racing racers, brings to the concept of sense data. It is the opportunity to overcome, without abandoning the idea of mental states, the physicalist criticisms about the concept. The world of experience is a world composed of tensors as sense data, as is our quantum universe that surrounds us.

\footnotetext{
${ }^{11}$ Huemer (2011).
} 